\title{
UNVEILING NEUTRINO MIXING AND LEPTONIC CP VIOLATION
}

\author{
OLGA MENA \\ Fermilab National Accelerator Laboratory \\ P.O. Box 500, Batavia, IL 60510 US \\ omena@fnal.gov \\ Received (Day Month Year) \\ Revised (Day Month Year)
}

\begin{abstract}
We review the present understanding of neutrino masses and mixings, discussing what are the unknowns in the three family oscillation scenario. Despite the anticipated success coming from the planned long baseline neutrino experiments in unraveling the leptonic mixing sector, there are two important unknowns which may remain obscure: the mixing angle $\theta_{13}$ and the CP-phase $\delta$. The measurement of these two parameters has led us to consider the combination of superbeams and neutrino factories as the key to unveil the neutrino oscillation picture.
\end{abstract}

Keywords: Neutrino oscillations, CP violation, degeneracies.

PACS Nos.: $14.60 \mathrm{Pq}$

\section{Introduction: status of neutrino masses and mixing, and pending questions.}

Neutrino oscillations have been observed and robustly established by the data from solar 12, atmospheric $\frac{3}{3}$, reactor 4 and long-baseline neutrino experiments $\underline{[5}$. Barring exotic interactions, these results indicate the existence of non-zero neutrino masses and mixings. The most economical, trivial way to accommodate these new parameters is via the three neutrino PMNS mixing matrix ${ }^{a}$, the leptonic analogue to the CKM matrix in the quark sector. Neutrino oscillations within this scenario are described by six parameters: two mass squared differences ${ }^{\mathrm{b}}\left(\Delta m_{12}^{2}\right.$ and $\left.\Delta m_{23}^{2}\right)$, three Euler angles $\left(\theta_{12}, \theta_{23}\right.$ and $\left.\theta_{13}\right)$ and one Dirac CP phase $\delta$. The standard way to connect the solar, atmospheric, reactor and accelerator data with some of the six oscillation parameters listed above is to identify the two mass splittings and the two mixing angles which drive the solar and atmospheric transitions with $\left(\Delta m_{12}^{2}\right.$,

${ }^{a}$ We restrict ourselves to a three-family neutrino scenario analysis. The unconfirmed LSND signal cannot be explained in terms of neutrino oscillations within this scenario, but might require additional light sterile neutrinos or more exotic explanations 6] The ongoing MiniBooNE experiment 7 is expected to explore all of the LSND oscillation parameter space 8 .

${ }^{\mathrm{b}} \Delta m_{i j}^{2} \equiv m_{j}^{2}-m_{i}^{2}$ throughout the paper. 


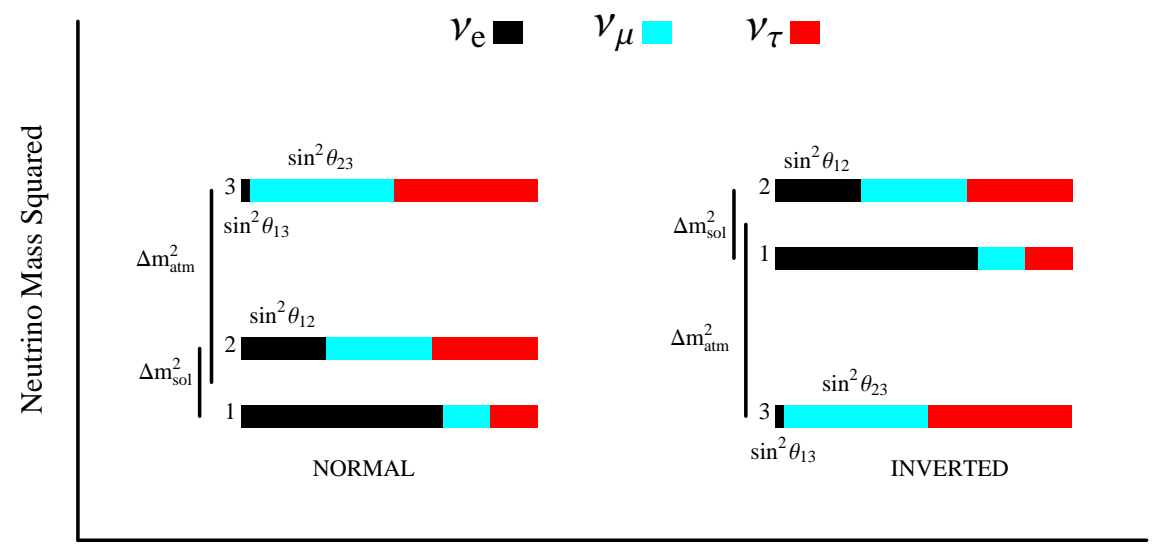

Fractional Flavor Content

Fig. 1. The range of probability of finding the $\alpha$-flavor in the $i$-the mass eigenstate as indicated for the two different mass hierarchies for the present best fit values of the mixing parameters.

$\left.\theta_{12}\right)$ and $\left(\left|\Delta m_{23}^{2}\right|, \theta_{23}\right)$, respectively. The sign of the splitting of the atmospheric state $\Delta m_{23}^{2}$ with respect to the solar doublet is one of the unknowns within the neutrino sector. Consequently, the mass eigenstates $\nu_{1}$ and $\nu_{2}$ involved in the solar doublet could have smaller mass than the third mass eigenstate $\nu_{3}$ (normal hierarchy) or larger mass than the former doublet (inverted hierarchy). Both possibilities are illustrated in Fig. 1 extracted from Ref 9 . The best fit point for the combined analysis of solar neutrino data together with KamLAND reactor data ${ }^{10}$ is at $\Delta m_{12}^{2}=8.2 \times 10^{-5} \mathrm{eV}^{2}$ and $\tan ^{2} \theta_{12}=0.4$. In the atmospheric neutrino sector, the most recent analysis of $\mathrm{K} 2 \mathrm{~K}$ accelerator neutrino data and atmospheric neutrino data 11 finds the best fit at $\left|\Delta m_{23}^{2}\right|=2.7 \times 10^{-3} \mathrm{eV}^{2}$ and $\sin ^{2} 2 \theta_{23}=1$.

The mixing angle $\theta_{13}$ (which connects the solar and atmospheric neutrino realms) and the amount $\mathrm{CP}$ violation in the leptonic sector are undetermined. At present, the upper bound on the angle $\theta_{13}$ coming from $\mathrm{CHOOZ}$ reactor neutrino data 12 is $\sin ^{2} 2 \theta_{13}<0.1$ (at $90 \%$ CL) for a value of the atmospheric mass gap close to the best fit reported before. The CP-phase $\delta$ is unobservable in current neutrino oscillation experiments. The experimental discovery of the existence of $\mathrm{CP}$ violation in the leptonic sector, together with the discovery of the Majorana neutrino character would point to leptogenesis as the source for the baryon asymmetry of the universe, provided that accidental cancellations are not present.

Several oscillation experiments that exploit neutrino beams from nuclear reactors and accelerators are taking data, and similar experiments will take data over the next few years. All of them have inaugurated a precision era in neutrino physics. 
Despite the anticipated progress coming from short-term future facilities, there are fundamental issues that will remain unknown, namely, in the oscillation picture:

- The value of $\theta_{13}$. Apart from the measurements of $\theta_{13}$ coming from future long baseline experiments, future reactor neutrino oscillation experiments could set the value of $\theta_{13}$, as explored in detail through Ref 13. Also, if neutrinos from astrophysical sources decay 14 , the deviations of the flavor ratios with respect to the values expected in the standard oscillation scenario could allow a measurement of $\theta_{13}$ and $\delta$.

- The ordering of the mass spectrum.

- The existence of leptonic CP Violation.

Moreover, in spite of the bright expectations associated even with the longerterm neutrino oscillation experiments, two fundamental neutrino properties would still remain unknown: their nature (Dirac or Majorana) and the absolute value of their masses. Both are outstanding questions that would remain to be answered with experiments other than the ones measuring neutrino oscillations.

Direct information on the absolute scale of neutrino masses can be extracted from the kinematics of weak decays involving neutrinos in their final states. The present upper bound on the electron neutrino mass from tritium beta decay experiments is $2.2 \mathrm{eV}$ (95\% CL) 15. The authors of Ref 16 find an upper limit of $1.8 \mathrm{eV}$ at the $95 \% \mathrm{CL}$ after the combination of the data from Mainz and Troitsk experiments. In the future, the KATRIN experiment is expected to be sensitive to electron neutrino masses of $\sim 0.2 \mathrm{eV}$ at the $90 \% \mathrm{CL} 17$.

Neutrinoless double beta decay searches are, at present, the only experimental way to establish the neutrino character. This type of decay is only possible if neutrinos are Majorana particles. Experimentally, the lack of the observation of neutrinoless double beta decay processes so far provides an upper bound on the socalled "effective Majorana mass" of the electron neutrino within the range $0.3-1.0$ $\mathrm{eV} 18$.

Next generation of neutrinoless double beta decay experiments could provide a definitive answer to the question of whether neutrinos are Dirac or Majorana. For a complete discussion of the potential of these future experiments in measuring fundamental neutrino properties, see Ref 19 .

Cosmology provides the key to tackle the "bare" absolute scale of neutrino masses, since it is sensitive to the overall neutrino mass scale, free of mixing parameters or CP-violating phase dependencies.

As pointed out in Ref 20 , neutrinos with masses smaller than the temperature at the recombination era $(T \sim 0.3 \mathrm{eV})$ are still relativistic at the time of last scattering, and their effect in terms of $\mathrm{CMB}$ perturbations is equivalent to the one present in the massless neutrino situation. If the neutrino mass is close to $0.3 \mathrm{eV}$, the free streaming scale is imprinted in the perturbations which enter inside the Hubble radius before decoupling. Perturbations smaller than the free streaming scale are therefore suppressed, leading to a suppression of of the matter power 
spectrum proportional to the ratio of the neutrino energy density to the overall matter density.

While CMB data alone does not provide a competitive bound on neutrino mass ${ }^{\mathrm{c}}$ if compared with present terrestrial kinematic constraints, a stringent bound on the sum of neutrino masses of about $\sum_{i} m_{\nu_{i}} \leq 1 \mathrm{eV}$ can be obtained by combining CMB data with large scale structure from 2df or SDSS 21]. The upper limit on $\sum_{i} m_{\nu_{i}}$ that can be achieved with cosmology depends somewhat on the priors and the data exploited in the analysis. For example, a recent joint analysis of CMB + $\mathrm{SN}-\mathrm{Ia}+\mathrm{HST}+\mathrm{LSS}$ data 16 provides a $2 \sigma$ bound on $\sum_{i} m_{\nu_{i}} \leq 1.4 \mathrm{eV}^{\mathrm{d}}$.

The determination of the fermion masses and mixings parameters is a mandatory first step that is essential for an understanding of the origin of flavor. Furthermore, as mentioned before, neutrino masses point to leptogenesis as the source of the matterantimatter asymmetry of the universe, provided $\mathrm{CP}$ is violated in the leptonic sector. - In the present study we concentrate on the determination of neutrino oscillation parameters and on the measurement of leptonic CP violation with long-baseline, accelerator-based neutrino oscillation experiments.

In particular, in the next sections we will explore in detail the possible measurement of the two unknown parameters $\theta_{13}$ and $\delta$ with future superbeam (SB) and neutrino factory $(\mathrm{NF})$ facilities, as these two designs appear to be among the most promising ways to unveil neutrino mixing and leptonic $\mathrm{CP}$ violation ${ }^{\mathrm{e}}$.

What is a Superbeam (SB) experiment 232425] It consists, basically, of a higher intensity version of a conventional neutrino (antineutrino) beam. Superbeams represent the logical next step in accelerator-based neutrino physics. A conventional neutrino beam is produced by a primary proton beam which hits a target and creates secondary beams of charged pions and kaons. If positively charged pions and kaons have been focused and directed into the decay channel, the resulting beam will contain mostly muon neutrinos produced in the two body decays $\pi^{+} \rightarrow \mu^{+} \nu_{\mu}$ and $K^{+} \rightarrow \mu^{+} \nu_{\mu}$. In the present work we exploit the experimental setup presented in Ref ${ }^{25}$, i.e, the CERN SPL superbeam with a 400 Kton Water Cherenkov detector ${ }^{\mathrm{f}}$ located at a $130 \mathrm{~km}$ distance from the neutrino source, in the Fréjus tunnel. We have assumed a 2 year-long run with $\pi^{+}$focusing, and a 8 year-long run with $\pi^{-}$focusing. The fluxes and detector systematics have been discussed in Ref 25 .

\footnotetext{
${ }^{\mathrm{c}}$ The free streaming scale for a neutrino mass $\sim \mathrm{eV}$ is smaller than the ones which can be probed using present CMB data.

${ }^{\mathrm{d}}$ If Ly $\alpha$ Forest is added as additional information in the SDSS data, the previous bound is improved by a factor of $\sim 3$. However, the systematics of the Ly $\alpha$ analysis is unclear and it has to be further explored.

eThe prospects of future measurements of these two oscillation parameters at different $\beta$ beams setups has been recently explored in Ref 22 .

${ }^{\mathrm{f}}$ It may be argued that such a detector may be unrealistic in practice. We shall use it with the purpose of illustrating the far-future physics perspective.
} 
We have not exploited the energy dependence of the signal because the neutrino spectrum is peaked at $\sim 0.25 \mathrm{GeV}$, and at such low energies the neutrino energy can not be reconstructed due to Fermi motion of nucleons inside the nuclear target (oxygen, in this case).

What is a Neutrino Factory (NF) 2627282930? It consists, essentially, of a muon storage ringg with long straight sections along which the muons decay. These muons provide high intensity neutrino beams (the neutrino flux is approximately $10^{4}$ times the flux of existing neutrino beams), which have a precisely-known neutrino flavor content, making them extremely superior to the conventional beams. Hence, compared to conventional neutrino beams from pion decay, the neutrino factory provides $\nu_{e}$ and $\bar{\nu}_{e}$ beams in addition to $\nu_{\mu}$ and $\bar{\nu}_{\mu}$ beams, with minimal systematic uncertainties on the neutrino flux and spectrum 31 . What is a "wrong sign muon" event 32 ? Suppose, for example, that positive charged muons have been stored in the ring. These muons will decay as $\mu^{+} \rightarrow e^{+}+\nu_{e}+\bar{\nu}_{\mu}$. The muon antineutrinos will interact in the detector to produce positive muons. Then, any "wrong sign muons" (negatively-charged muons) detected are an unambiguous proof of electron neutrino oscillations, in the $\nu_{e} \rightarrow \nu_{\mu}$ channel. This is, precisely, the golden channel, which will be shown to be essential for the goals of the present study. We will consider a neutrino factory providing $10^{21} \mu^{+}$and $\mu^{-}$of $50 \mathrm{GeV}$ and a $40 \mathrm{kton}$ iron magnetized calorimeter detector. We have exploited the dependence of the signal in the neutrino energy assuming 5 energy bins 32 . Hereafter, we will refer to this setup as the standard setup.

The appearance of "wrong-sign muons" at three reference baselines $(732 \mathrm{Km}$, $2810 \mathrm{Km}$ and $7332 \mathrm{~km}$ ) has been considered. Realistic background and efficiencies for the proposed 40 kton iron magnetized calorimeter detector, see Ref 33, as well as accurate matter effects along the neutrino path, have been included in our numerical analysis as well 32 .

\section{Measurement of $\theta_{13}$ and $\mathrm{CP}-\delta$ at a neutrino factory through the golden channels.}

In this section we will show that the most promising way to determine the unknown parameters $\delta$ and $\theta_{13}$ is through the detection of the subleading transitions $\nu_{e} \rightarrow \nu_{\mu}$ and $\bar{\nu}_{e} \rightarrow \bar{\nu}_{\mu}$ by using the golden signature of wrong sign muons 32. Defining

$\Delta_{i j} \equiv \frac{\Delta m_{i j}^{2}}{2 E}$, a convenient and precise approximation is obtained by expanding to second order in the following small parameters: $\theta_{13}, \Delta_{12} / \Delta_{23}, \Delta_{12} / A$ and $\Delta_{12} L$. The result is (details of the calculation can be found in Ref $\underline{32}$ ):

$P_{\nu_{e} \nu_{\mu}\left(\bar{\nu}_{e} \bar{\nu}_{\mu}\right)}=s_{23}^{2} \sin ^{2} 2 \theta_{13}\left(\frac{\Delta_{23}}{\tilde{B}_{\mp}}\right)^{2} \sin ^{2}\left(\frac{\tilde{B}_{\mp} L}{2}\right)+c_{23}^{2} \sin ^{2} 2 \theta_{12}\left(\frac{\Delta_{12}}{A}\right)^{2} \sin ^{2}\left(\frac{A L}{2}\right)$

${ }^{\mathrm{g}}$ This muon storage ring is an essential stepping-stone towards possible muon colliders. 


$$
+\tilde{J} \frac{\Delta_{12}}{A} \frac{\Delta_{23}}{\tilde{B}_{\mp}} \sin \left(\frac{A L}{2}\right) \sin \left(\frac{\tilde{B}_{\mp} L}{2}\right) \cos \left( \pm \delta-\frac{\Delta_{23} L}{2}\right),
$$

where $L$ is the baseline, $\tilde{B}_{\mp} \equiv\left|A \mp \Delta_{23}\right|$ and the matter parameter $A$ is defined in terms of the average electron number density, $n_{e}(L)$, as $A \equiv \sqrt{2} G_{F} n_{e}(L)$, where the $L$-dependence will be taken from $\operatorname{Ref} 34$. The $\tilde{J}$ parameter is defined as

$$
\tilde{J} \equiv \cos \theta_{13} \sin 2 \theta_{13} \sin 2 \theta_{23} \sin 2 \theta_{12} .
$$

After a careful exploration of the energy and baseline dependence of the different terms in Eq. (11) 32, and a detailed study of the CP-asymmetry as defined in Ref 28323513637 .

$$
\bar{A}_{e \mu}^{C P}=\frac{\left\{N\left[\mu^{-}\right] / N_{o}\left[e^{-}\right]\right\}_{+}-\left\{N\left[\mu^{+}\right] / N_{o}\left[e^{+}\right]\right\}_{-}}{\left\{N\left[\mu^{-}\right] / N_{o}\left[e^{-}\right]\right\}_{+}+\left\{N\left[\mu^{+}\right] / N_{o}\left[e^{+}\right]\right\}_{-}}
$$

and after substracting the fake $\mathrm{CP}$ violating effects induced by matter, it turns out that the optimal distance to be sensitive to the CP-phase $\delta$ at a future NF exploiting muons with energies $E_{\mu}=50 \mathrm{GeV}$ is $\mathcal{O}(3000) \mathrm{Km} 32$.

In the limit $A \rightarrow 0$, the expression Eq. (11) reduces to the simple formulae in vacuum 32

$$
\begin{aligned}
P_{\nu_{e} \nu_{\mu}\left(\bar{\nu}_{e} \bar{\nu}_{\mu}\right)} & =s_{23}^{2} \sin ^{2} 2 \theta_{13} \sin ^{2}\left(\frac{\Delta_{23} L}{2}\right)+c_{23}^{2} \sin ^{2} 2 \theta_{12} \sin ^{2}\left(\frac{\Delta_{12} L}{2}\right) \\
& +\tilde{J} \cos \left( \pm \delta-\frac{\Delta_{23} L}{2}\right) \frac{\Delta_{12} L}{2} \sin \left(\frac{\Delta_{23} L}{2}\right) .
\end{aligned}
$$

As in Ref 38139, we will denote the three terms in Eq. (4), atmospheric, solar and interference, by $P^{a t m}, P^{s o l}$ and $P_{\nu(\bar{\nu})}^{\text {inter }}$, respectively. When $\theta_{13}$ is relatively large, the probability is dominated by the atmospheric term. We will refer to this situation as the atmospheric regime. Conversely, when $\theta_{13}$ is very small, the solar term dominates $P^{s o l} \gg P_{\nu(\bar{\nu})}^{a t m}$. This is the solar regime. The interference term is the only one which contains the $\mathrm{CP}$ phase $\delta$, and it is the only one which differs for neutrinos and antineutrinos. The vacuum approximation Eq. (44) should be excellent in the SB scenario with a baseline of a few hundreds of kilometers, while in practice it also gives a good indication for the results at the short $(732 \mathrm{~km})$ and intermediate $(2000-3000 \mathrm{~km})$ baselines of a NF.

The next step is to perform an exhaustive numerical treatment. All numerical results simulated have been obtained with the exact formulae for the oscillation probabilities 323839 . We thus performed $\chi^{2}$ fit analysis to the simultaneous extraction of $\delta$ and $\theta_{13}$ at a future neutrino factory complex assuming the standard setup described in the previous section. Unless specified otherwise, we take the following central values for the remaining oscillation parameters: $\sin ^{2} 2 \theta_{12} \cdot \Delta m_{12}^{2}=1 \times 10^{-4}$ $\mathrm{eV}^{2},\left|\Delta m_{23}^{2}\right|=2.5 \times 10^{-3} \mathrm{eV}^{2}$ and $\sin ^{2} 2 \theta_{23}=1$. The $\chi^{2}$ for a fixed baseline $\lambda$ is defined as:

$$
\chi_{\lambda}^{2}=\sum_{i, j} \sum_{p, p^{\prime}}\left(n_{i, p}^{\lambda}-N_{i, p}^{\lambda}\right) C_{i, p:, j, p^{\prime}}^{-1}\left(n_{j, p^{\prime}}^{\lambda}-N_{j, p^{\prime}}^{\lambda}\right)
$$


where $N_{i, \pm}^{\lambda}$ is the predicted number of wrong-sign muons for a certain oscillation hypothesis, $n_{i, p}^{\lambda}$ are the simulated "data" from a Gaussian or Poisson smearing and $C$ is the $2 N_{\text {bin }} \times 2 N_{\text {bin }}$ covariance matrix, that for this illustration only contains statistical errors. We can safely neglect the effects on the fit induced by the uncertainties in the remaining oscillation parameters. The impact of the projected uncertainties on the knowledge of the solar and atmospheric parameters and on the matter density at the time of the NF has been considered, and was found that these uncertainties do not significantly affect the global fits 38 . In Fig. 2 we show the $68.5 \%, 90 \%$ and $99 \%$ contours for a $\chi^{2}$ fit to the data from a future NF with the detector of Ref 33 located at a baseline $L=2810 \mathrm{~km}$. The "true" parameter values that we have chosen for this example are depicted in the figure with a star, that is, $\delta=54^{\circ}$ and $\theta_{13}=2^{\circ}$.

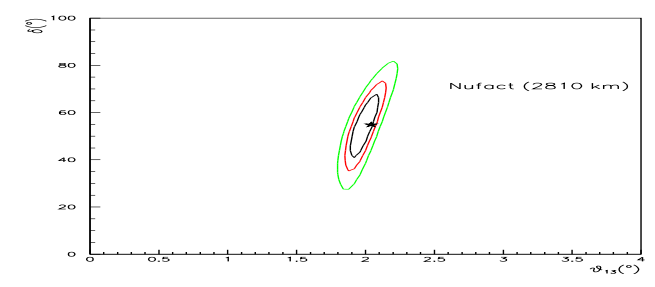

Fig. 2. $68.5 \%, 90 \%$ and $99 \%$ contours resulting from the fits at $L=2810 \mathrm{~km}$ for $\delta=54^{\circ}$ and $\theta_{13}=2^{\circ}$.

By observing the results presented in Fig. 2 it seems that it is possible to extract simultaneously $\theta_{13}$ and $\delta$ with very good precision at a future NF with the standard setup and the far detector located at distances $\sim 3000 \mathrm{Km}$. However, as the reader can notice, in this example we only have considered a limited CP-phase $\delta$ range $\left(0^{\circ}<\delta<90^{\circ}\right)$, while the full parameter range $-180^{\circ}<\delta<180^{\circ}$ should be explored.

\section{Degenerate solutions.}

We can ask ourselves whether it is possible to unambiguously determine $\delta$ and $\theta_{13}$ by measuring the transition probabilities $\nu_{e} \rightarrow \nu_{\mu}$ and $\bar{\nu}_{e} \rightarrow \bar{\nu}_{\mu}$ at fixed neutrino energy and at just one NF baseline. The answer is no. By exploring the full (allowed) range of the $\delta$ and $\theta_{13}$ parameters, that is, $-180^{\circ}<\delta<180^{\circ}$ and $0^{\circ}<\theta_{13}<10^{\circ}$, we find, at fixed neutrino energy and at fixed baseline, the existence of degenerate solutions $\left(\delta^{\prime}, \theta_{13}^{\prime}\right)$, that we label intrinsic degeneracies, which give the same oscillation probabilities than the set $\left(\delta, \theta_{13}\right)$ chosen by Nature 38 . More explicitly, if $\left(\theta_{13}\right.$, $\delta)$ are the values chosen by nature, the conditions 
8 Unveiling neutrino mixing

$$
\left.\begin{array}{l}
P_{\nu_{e} \nu_{\mu}}\left(\theta_{13}^{\prime}, \delta^{\prime}\right)=P_{\nu_{e} \nu_{\mu}}\left(\theta_{13}, \delta\right) \\
P_{\bar{\nu}_{e} \bar{\nu}_{\mu}}\left(\theta_{13}^{\prime}, \delta^{\prime}\right)=P_{\bar{\nu}_{e} \bar{\nu}_{\mu}}\left(\theta_{13}, \delta\right)
\end{array}\right\}
$$

can be generically satisfied by another set $\left(\theta_{13}^{\prime}, \delta^{\prime}\right)$. It has also been pointed out that other fake solutions might appear from unresolved degeneracies in two other oscillation parameters:

(1) At the time of the future NF, the sign of the atmospheric mass difference $\Delta m_{23}^{2}$ may remain unknown, that is, we would not know if the hierarchy of the neutrino mass spectrum is normal or inverted. In this particular case, $P\left(\theta_{13}^{\prime}, \delta^{\prime},-\Delta m_{23}^{2}\right)=P\left(\theta_{13}, \delta, \Delta m_{23}^{2}\right) 4041$

(2) Disappearance experiments only give us information on $\sin ^{2} 2 \theta_{23}$ : is $\theta_{23}$ in the first octant, or is it in the second one, $\left(\pi / 2-\theta_{23}\right)$ ?. In terms of the probabilities, $P\left(\theta_{13}^{\prime}, \delta^{\prime}, \frac{\pi}{2}-\theta_{23}\right)=P\left(\theta_{13}, \delta, \theta_{23}\right) 4142$.

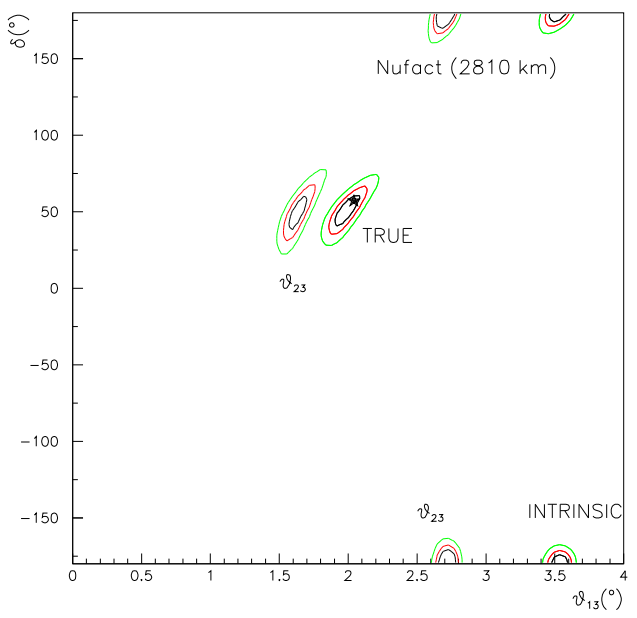

Fig. 3. $68.5 \%, 90 \%$ and $99 \%$ contours resulting from the fits at $L=2810 \mathrm{~km}$ for $\delta=54^{\circ}$ and $\theta_{13}=2^{\circ}$. Three fake solutions appear, in addition to the true one. The degeneracy corresponding to the case of a global fit to data with the wrong choice of the sign of $\Delta m_{23}^{2}$ and of the $\theta_{23}$ octant is not depicted.

All these ambiguities complicate the experimental determination of $\delta$ and $\theta_{13}$. To illustrate in brief the degeneracy problem, imagine as an example that the values chosen by Nature are $\delta=54^{\circ}$ and $\theta_{13}=2^{\circ}$, and consider the NF fluxes with a detector of the type discussed in Ref 33 located at $L=2810 \mathrm{~km}$. A global fit of the experimental data using the spectral information would result in a cluster of 
solutions some of which are depicted in the Fig. 3 A constellation of fake solutions $\left(\theta_{13}^{\prime}, \delta^{\prime}\right)$ surrounds the true one: those induced by the intrinsic degeneracy and by the $\theta_{23}$ octant ambiguity are shown, whereas those coming from the ambiguity in the sign of $\Delta m_{23}^{2}$ are absent for the particular case analyzed here due to the presence of sizable matter effects (as it is well known, if the atmospheric mass splitting is positive, i.e, normal spectrum, the oscillation probabilities of neutrinos (antineutrinos) is enhanced (depleted)).

Finally, the combined degeneracy that would arise when performing a fit with both the wrong choices of the $\operatorname{sign}\left(\Delta m_{23}^{2}\right)$ and of the $\theta_{23}$ octant is not depicted. From the results shown in Fig. 31 we see that we would not be able to determine whether $\delta \simeq 54^{\circ}$ ( $\mathrm{CP}$ is violated $)$ or $\delta \simeq 180^{\circ}$ ( $\mathrm{CP}$ is conserved)!

\section{Resolving the degeneracies}

In Ref 32 it was pointed out that some of the degeneracies listed above could be eliminated with sufficient energy or baseline spectral information. In practice, however, the spectral information has been shown to be not strong enough to resolve degeneracies with a single detector, once that statistical errors and realistic efficiencies and backgrounds are taken into account. A lot of work has been thus devoted to resolve the degeneracies by exploiting the different neutrino energy and baseline dependence of two (or more) LBL experiments. For instance, it has been suggested in Ref 38 to combine the results of the optimal NF baseline $(\mathcal{O}(3000) \mathrm{km})$, with other $\mathrm{NF}$ baselines ${ }^{\mathrm{h}}$. An independent appearance channel at future neutrino factories, the so called silver channels $44455 \nu_{e} \rightarrow \nu_{\tau}$ and $\bar{\nu}_{e} \rightarrow \bar{\nu}_{\tau}$, can resolve the intrinsic degeneracies, provided that $\theta_{13}>1^{\circ}$. We will exploit the potential of the silver channels in the next sections. A higher gamma beta beam has been recently proposed and the potential of such a novel technique has been shown to be competitive with the NF 22. For a recent theoretical study of the parameter degeneracies, and the perspectives for resolving them through the combination of data from NF, SB and/or beta beam, see Ref 46 . The physics potential of the combination of future beta beams and SB facilities has been carefully analyzed in Ref 47 .

If the value of $\theta_{13}$ turns out to be not very small, combinations of future long baseline experiments can also help enormously 48495051]. Another possibility is to exploit the data from neutrino reactor experiments, one of the best ways to deal

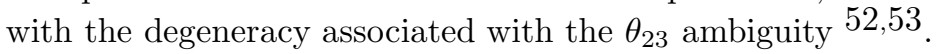

\subsection{Exploiting the different $E, L$ of superbeam experiments}

Using the approximate formulae of Eq. (4), it is easy to find the expression for the

intrinsic degeneracies in the atmospheric and solar regimes. In Ref 38 the general results including matter effects are given. Solutions in vacuum are derived in Ref 39 .

hIf lower energy threshold detectors are a viable option, the degeneracies can be resolved at a single NF baseline 43 . 
These solutions are easier to understand and are a good approximation for the baselines relevant to neutrino superbeams. In the present study we review in detail the case of the intrinsic degeneracies. We then summarize the situation for the sign and $\theta_{23}$-octant degeneracies, by noticing that there exists a common pattern in these fake solutions.

For instance, we show that for $\theta_{13}$ sufficiently large and in the vacuum approximation, apart from the true solution, there is a fake one at 38139

$$
\begin{aligned}
\delta^{\prime} & \simeq \pi-\delta, \\
\theta_{13}^{\prime} & \simeq \theta_{13}+\cos \delta \sin 2 \theta_{12} \frac{\Delta m_{12}^{2} L}{4 E} \cot \theta_{23} \cot \left(\frac{\Delta m_{23}^{2} L}{4 E}\right) .
\end{aligned}
$$

Note that for the values $\delta=-90^{\circ}, 90^{\circ}$, the fake solution disappears. Typically, $\cot \left(\frac{\Delta m_{23}^{2} L}{4 E}\right)$ has on average opposite sign for the proposed SB and NF setups ${ }^{\mathrm{i}}$, for $\Delta m_{23}^{2}=0.003 \mathrm{eV}^{2}$, as can be seen in Table 1

Table 1. Parameters for possible superbeam, neutrino factory, and $\beta$-beam setups.

\begin{tabular}{cccc}
\hline & $\langle E\rangle(\mathrm{GeV})$ & $\mathrm{L}(\mathrm{km})$ & $\cot \left(\frac{\Delta m_{23}^{2} L}{4 E}\right)$ \\
\hline SB-SPL & 0.25 & 130 & -0.43 \\
JHF-off-axis & 0.7 & 295 & -0.03 \\
NF@732 & 30 & 732 & +10.7 \\
NF@2810 & 30 & 2810 & +2.68 \\
$\beta$-beam & 0.35 & 130 & +0.17 \\
\hline
\end{tabular}

When $\theta_{13} \rightarrow 0$ and in the vacuum approximation, the intrinsic degeneracy is independent of $\delta 3839$.

$$
\left.\begin{array}{l}
\text { if } \cot \left(\frac{\Delta m_{23}^{2} L}{4 E}\right)>0 \text { then } \delta^{\prime} \simeq \pi \\
\text { if } \cot \left(\frac{\Delta m_{23}^{2} L}{4 E}\right)<0 \text { then } \delta^{\prime} \simeq 0
\end{array}\right\} \quad \theta_{13}^{\prime} \simeq \sin 2 \theta_{12} \frac{\Delta m_{12}^{2} L}{4 E}\left|\cot \theta_{23} \cot \left(\frac{\Delta m_{23}^{2} L}{4 E}\right)\right| \text {. }
$$

This solution is referred to in the literature as the $\theta_{13}=0$-mimicking solution, and occurs because there is a value of $\theta_{13}^{\prime}$ for which there is an exact cancellation of the atmospheric and interference terms in both the neutrino and antineutrino probabilities simultaneously, with $\sin \delta^{\prime}=0$ 3839.

The $\theta_{13}^{\prime}-\theta_{13}$ difference of the fake solution depends strongly on the baseline and the neutrino energy through the ratio $L / E$, so the combination of the results of two experiments with a different value for this ratio should be able to resolve these degeneracies. Even more important is that, for small $\theta_{13}, \delta^{\prime}$ may differ by $180^{\circ}$ if the two facilities have opposite sign for $\cot \left(\frac{\Delta m_{23}^{2} L}{4 E}\right)$, see Eqs. (8). We note

${ }^{\mathrm{i}}$ Clearly the parameters for these setups are not fixed yet and might be modified conveniently in the final designs. 
that $\cot \left(\frac{\Delta m_{23}^{2} L}{4 E}\right)=0$ at the maximum of atmospheric neutrino oscillations in vacuum. For the NF setups, the sign of $\cot \left(\frac{\Delta m_{23}^{2} L}{4 E}\right)$ is clearly positive (see Tab. (1), since the measurement of $\mathrm{CP}$ violation requires, because of the large matter effects, a baseline considerably shorter than that corresponding to the maximum of the atmospheric oscillation (in vacuum). In the SB scenario on the other hand, because of the smaller $\langle E\rangle$, matter effects are small at the maximum of the atmospheric oscillation, which then becomes the optimal baseline for $\mathrm{CP}$ violation studies, and therefore $\cot \left(\frac{\Delta m_{23}^{2} L}{4 E}\right)$ should be chosen close to zero. It is then not very difficult to ensure that $\cot \left(\frac{\Delta m_{23}^{2} L}{4 E}\right)$ be dominantly negative in this case ${ }^{\mathrm{j}}$, which results in an optimal complementarity of the two facilities in resolving degeneracies.

Although the combination of the data from two SB facilities with different $E / L \sim \Delta m_{23}^{2}$ could also a priori overcome the degeneracies, SB projects are in general planned to exploit data on or nearby the atmospheric oscillation maximum and, therefore, the differences in their $E / L$ are not large enough to fully resolve the degeneracies.

A detailed combined analysis of the results from a neutrino factory 3238 and those from a superbeam 23 facility has also been performed. The combination of superbeams with the optimal NF baseline of $L=2810 \mathrm{~km}$ has been shown 38 to be sufficient to eliminate all the fake solutions even in the solar regime. For the sake of illustration, consider the values $\delta=54^{\circ}$ and $\theta_{13}=2^{\circ}$. After combining the data obtained at a NF baseline of $L=2810 \mathrm{~km}$ and the data from the SPL-SB facility, the fit clearly selects the correct solution, see Fig. 4.

Evidently, this example is for a rather high value of $\theta_{13}$, that is $\theta_{13}=2^{\circ}$. The parameter space down to very low values of $\theta_{13}$ is explored in detail in Ref ${ }^{[39}$, where it is shown that the fake solutions associated with the sign of the atmospheric mass difference and $\theta_{23}$ ambiguities can be grouped in two sets: those close to nature's true values (solutions of type I) and those related to the intrinsic fake solution (solutions of type II). Generically solutions of type I show a milder $L / E$ dependence and are thus more difficult to eliminate through the combination of the data from the two facilities considered here. We study all the fake solutions both analytically and through numerical simulations, and here we summarize the expected results after considering both experiments:

- The intrinsic degeneracies disappear after the $\mathrm{NF}+\mathrm{SB}$ combination down to the

${ }^{\mathrm{j} N o t e}$ however that most neutrino beams are generally wide-band beams in energy so it is necessary for this argument to hold that most of the interactions originate from neutrinos with an energy giving the appropriate $\cot \left(\frac{\Delta m_{23}^{2} L}{4 E}\right)$ sign. The results of the fits performed in Ref 39 indicate that this is the case in the two facilities (NF and SPL-SB) that are considered in detail in this paper. 


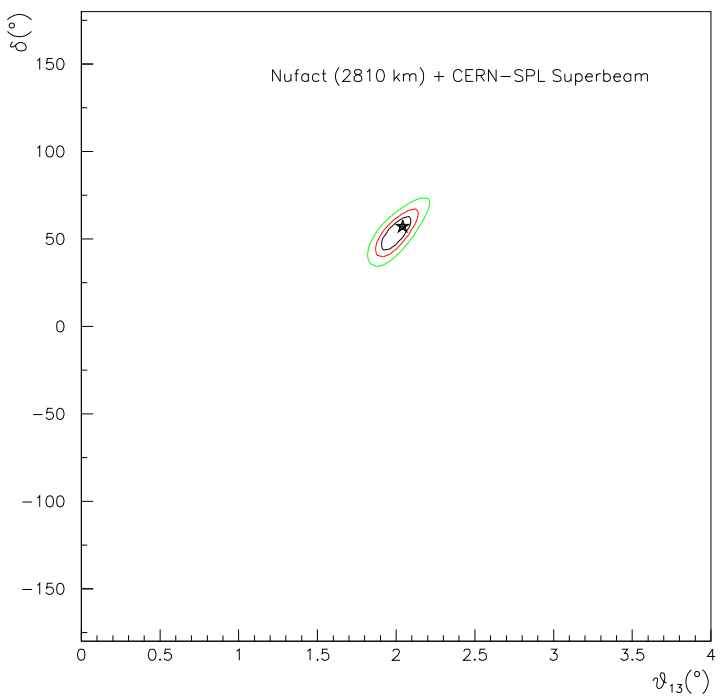

Fig. 4. Fits combining the results from the SPL-SB facility and from a neutrino factory baseline at $L=2810 \mathrm{~km}$ for $\delta=54^{\circ}$ and $\theta_{13}=2^{\circ}$. Notice that the fake intrinsic solutions have completely disappeared at the $3 \sigma C L$ in the combination.

sensitivity limit, for all three reference NF baselines considered here. If the other degeneracies are not considered, the sensitivity limit is $\theta_{13} \sim 0.3^{\circ}$ for a medium baseline NF $(L=2810 \mathrm{~km})$ and $\theta_{13} \sim 0.6^{\circ}$ for a short baseline NF $(L=732$ $\mathrm{km})$. Although this short distance is below $\sim \mathcal{O}(1000) \mathrm{km}$, and therefore it is not sensitive to $\mathrm{CP}$-Violation effects $^{\mathrm{k}}$, it is a very interesting distance after combining its results from those from the SPL-SB facility.

- The degeneracies that arise due to the $\operatorname{sign}\left(\Delta m_{23}^{2}\right)$ ambiguity can be resolved by combining the results from a NF with $L=2810 \mathrm{~km}$ and those from the SPL-SB facilities for $\theta_{13} \geq 1^{\circ}$. For shorter baselines $(L=732 \mathrm{~km})$ these fake solutions can be resolved after the combination for values of $\theta_{13}$ near its upper present bound of $\theta_{13}<10^{\circ}$, given by $\mathrm{CHOOZ} 12$. At very small values of $\theta_{13}$, the sign of $\Delta m_{23}^{2}$ remains an ambiguity, but it does not interfere much with the determination of $\theta_{13}$ (in this particular case, $\theta_{13}^{\prime}=\theta_{13}$ ) and with the measurement of leptonic $\mathrm{CP}$ violation, since $\delta^{\prime}=180^{\circ}-\delta$. The former implies that $\sin \delta^{\prime}=\sin \delta$.

- The degeneracies due to the $\left(\theta_{23}, \pi / 2-\theta_{23}\right)$ ambiguity are difficult to resolve and they can interfere with the measurement of $\theta_{13}$ and $\delta$. The combination of NF and SB experiments helps enormously in minimizing the bias in the extraction

\footnotetext{
${ }^{\mathrm{k}}$ We are considering neutrino energies of several tens of $\mathrm{GeV}$.
} 
of $\theta_{13}$ and $\delta$, though. However, as we will explore in the next sections, the analysis of the silver channels can help in this respect.

\subsection{Exploiting the different channels at the neutrino factory}

In the previous section we have pointed out that the degeneracies associated with the $\theta_{23}$ octant ambiguity are not fully resolved after the combination of the data from NF and SB experiments. Particularly difficult to overcome are type I solutions ${ }^{1}$ since these solutions are nearly $L / E$ independent. However, as first noticed in Ref $\frac{44}{4}$, an additional measurement of an independent appearance channel, $\nu_{e} \rightarrow \nu_{\tau}$ and $\bar{\nu}_{e} \leftrightarrow \bar{\nu}_{\tau}$ (silver channels) greatly helps in resolving the fake solution of type I associated with the $\theta_{23}$-ambiguity, since the locations of the fake solutions that arise from the data analysis of silver and golden channels differ substantially.

Consider the approximate oscillation probabilities 3244 in vacuum for $\nu_{e} \rightarrow \nu_{\tau}$ $\left(\bar{\nu}_{e} \rightarrow \bar{\nu}_{\tau}\right)$ :

$$
\begin{aligned}
P_{\nu_{e} \nu_{\tau}\left(\bar{\nu}_{e} \bar{\nu}_{\tau}\right)} & =c_{23}^{2} \sin ^{2} 2 \theta_{13} \sin ^{2}\left(\frac{\Delta m_{23}^{2} L}{4 E}\right)+s_{23}^{2} \sin ^{2} 2 \theta_{12}\left(\frac{\Delta m_{12}^{2} L}{4 E}\right)^{2} \\
& -\tilde{J} \cos \left( \pm \delta-\frac{\Delta m_{23}^{2} L}{4 E}\right) \frac{\Delta m_{12}^{2} L}{4 E} \sin \frac{\Delta m_{23}^{2} L}{4 E}
\end{aligned}
$$

They differ from those in Eq. (4) by the interchange $\theta_{23} \rightarrow \pi / 2-\theta_{23}$ and by a change in the sign of the interference term. As a result, in the atmospheric regime, the location of the fake solutions related to the $\theta_{23}$ ambiguity is opposite in sign for $\nu_{e} \rightarrow \nu_{\tau}$ and $\bar{\nu}_{e} \rightarrow \bar{\nu}_{\tau}$ oscillations and for $\nu_{e} \rightarrow \nu_{\mu}$ and $\bar{\nu}_{e} \rightarrow \bar{\nu}_{\mu}$ oscillations. In the solar regime, on the other hand, the solution of type I for the $\nu_{\tau}$ appearance measurement coincides with the one for $\nu_{\mu}$ appearance, while solutions of type II are different, as shown in Ref 39 . For the analysis of the silver channel we assume the setup of the Opera proposal $\underline{54}$, with one fixed baseline, $732 \mathrm{~km}$, i.e. the distance from CERN to Gran Sasso Laboratories in Italy, and a 4 Kton lead plus emulsion detector with spectrometers. A dedicated analysis can be found in Ref 45. We have found that after the combination of the results from NF golden $(L=2810$ $\mathrm{km})$ and SB silver $(L=732 \mathrm{~km})$ channels no degeneracy related to the $\theta_{23}$-octant ambiguity survives for $\theta_{13}>0.6^{\circ}$, at least assuming an ideal detector. This limit is expected to become more stringent, namely, $\theta_{13}>1^{\circ}$, when adding detector efficiencies and backgrounds. At very small values of $\theta_{13}\left(\theta_{13}<0.6^{\circ}\right)$, the $\theta_{23^{-}}$ octant ambiguity remains, but it does not interfere with the extraction of the two unknown parameters, $\theta_{13}$ and $\delta$, due to the location of the fake solution: $\theta_{13}{ }^{\prime} \sim \theta_{13}$ and $\delta^{\prime} \sim \pi-\delta$.

\footnotetext{
${ }^{1}$ The location of this degeneracy can lie far apart from the true values. In particular, if the true value of $\delta$ is $\mathrm{CP}$-violating, this fake solution appears to be consistent with $\mathrm{CP}$-conservation.
} 


\section{Getting the most from the combination of measurements at neutrino factories and at superbeam experiments}

We have considered 55 the impact of three simultaneous experiments (see Fig. 5 ) with 2 years running in the $\pi^{+}$polarity and 10 years running in the $\pi^{-}$polarity 56 . Simulated data from golden channels in a SB experiment, and from golden and silver channels in a NF experiment, have been combined. As the first stage of a detailed study with a realistic experimental setup $\underline{[56]}$ we consider here an ideal situation, neglecting backgrounds and efficiencies for an emulsion cloud chamber (ECC) detector. In Fig. [6 we show preliminary results for the simultaneous measurement of $\theta_{13}$ and $\delta$ for true values $\theta_{13}=0.6^{\circ}$ and four possible values for the CP phase $\delta=90,0,-90$ and 180 degrees.

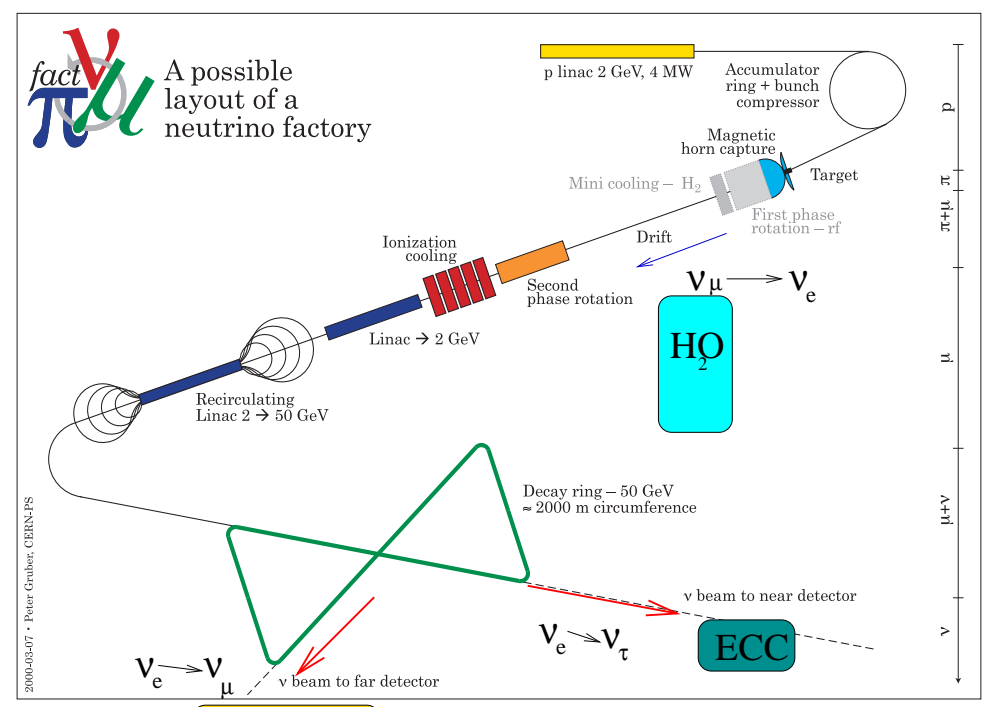

Fe detector

Fig. 5. The complex of NF (golden and silver channels) and the SPL-SB experiments.

\section{Conclusions}

It is a very interesting era for neutrino physics. New physics is revealed at a steady pace from a wealth of neutrino oscillation measurements. One of our major challenges is to search for a theoretical model to accommodate in a natural way the new physics scale that the observations suggest. A measurement of the absolute mass 


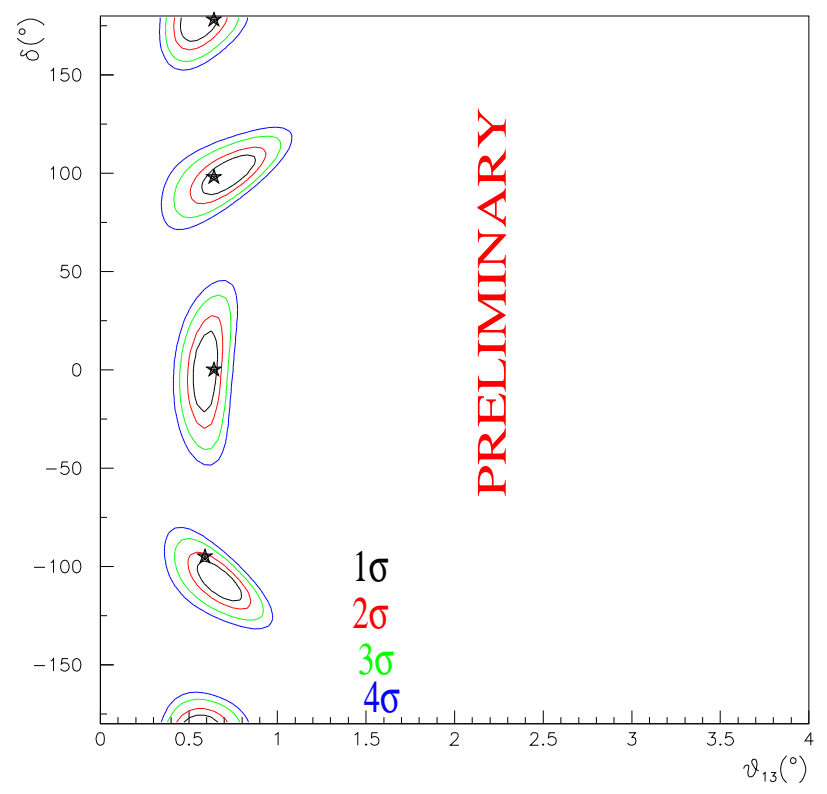

Fig. 6. Simultaneous extraction of $\delta$ and $\theta_{13}$ after the combination of three experiments described in the text.

for one neutrino would allow to guide us towards this new physics scale. Neutrino oscillation physics is already able to determine the mass differences and the mixing parameters between neutrino flavors and contribute potentially to the overall understanding of the origin of fermion masses. There exist several ongoing neutrino oscillation experiments which exploit solar, reactor, atmospheric and accelerator neutrinos and there are some planned experiments as well (mostly acceleratorbased). All of them have inaugurated a precision era in Neutrino Physics. Here we have focused in one of the major challenges ahead, that is the measurement of the angle $\theta_{13}$ and the CP-phase $\delta$. The difficulties associated with extracting these two parameters have led us to consider the potential of Superbeam (SB) and Neutrino Factory (NF) facilities.

Short-term superbeam and longer-term neutrino factory experiments can be viewed as sequential steps towards the same physics goals, and not as two alternative options. In this perspective, it is natural to combine their expected results. We have thus shown the enormous potential of combining the data from the SB and NF facilities with realistic setups, to eliminate the degeneracies in the simultaneous measurement of $\delta$ and $\theta_{13}$.

The only degeneracy that survives after the combination of SB and NF data is the one associated with the $\theta_{23}$ ambiguity. We have shown that it is possible to 
eliminate this degeneracy through the combination of NF golden and silver channels down to values of $\theta_{13} \sim 0.6^{\circ}$ in the present analysis, considering an ideal detector for the silver channel. A realistic experimental scenario is under study 56 .

It is very important to note that there exist several alternative experimental setups that could help enormously in disentangling the neutrino puzzle: Nova 57 , $\mathrm{T} 2 \mathrm{~K}[58$, reactor experiments and beta-beams facilities, whose potential is not discussed here.

All the latter experimental options have to be thoroughly explored in order to ascertain the ultimate precision in the determination of the detailed pattern of neutrino mass differences and mixing angles, a prerequisite to understand their origin and their relationship to the analogous parameters in the quark sector. The NF (golden and silver channels) plus its predecessor, the SB experiment, would provide the key to fulfill this goal.

\section{Acknowledgements}

The author would like to thank J. Burguet-Castell, A. Cervera, A. Donini, M. B. Gavela, J. Gómez Cádenas, P. Hernández and S. Rigolin for collaboration. It is a great pleasure to thank M. B. Gavela, S. Rigolin and M. Sorel for useful comments and suggestions on the manuscript. Fermilab is operated by URA under DOE contract DE-AC02-76CH03000.

\section{References}

1. Q. R. Ahmad et al. [SNO Collaboration], Phys. Rev. Lett. 89, 011301 (2002); Q. R. Ahmad et al. [SNO Collaboration], Phys. Rev. Lett. 89, 011302 (2002); S. N. Ahmed et al. [SNO Collaboration], Phys. Rev. Lett. 92, 181301 (2004).

2. S. Fukuda et al. [Super-Kamiokande Collaboration], Phys. Lett. B 539, 179 (2002).

3. Y. Fukuda et al. [Super-Kamiokande Collaboration], Phys. Rev. Lett. 81, 1562 (1998).

4. K. Eguchi et al. [KamLAND Collaboration], Phys. Rev. Lett. 90, 021802 (2003).

5. M. H. Ahn et al. [K2K Collaboration], Phys. Rev. Lett. 90, 041801 (2003).

6. G. Barenboim, L. Borissov, J. Lykken and A. Y. Smirnov, JHEP 0210, 001 (2002).

7. www-boone.fnal.gov/publicpages/runplan.ps.gz

8. A. Aguilar et al. [LSND Collaboration], Phys. Rev. D 64, 112007 (2001).

9. O. Mena and S. J. Parke, Phys. Rev. D 69, 117301 (2004).

10. T. Araki et al. [KamLAND Collaboration], arXiv:hep-ex/0406035

11. E. Kearns, Atmospheric neutrino results from SuperKamiokande, and T. Nakaya, K2K results, talks given at Neutrino 2004 (Paris, France).

12. M. Apollonio et al. [CHOOZ Collaboration], Phys. Lett. B 466, 415 (1999).

13. K. Anderson et al., arXiv:hep-ex/0402041

14. J. F. Beacom, N. F. Bell, D. Hooper, S. Pakvasa and T. J. Weiler, Phys. Rev. D 69, 017303 (2004).

15. Y. Farzan and A. Y. Smirnov, Phys. Lett. B 557, 224 (2003); Y. Farzan, O. L. G. Peres and A. Y. Smirnov, Nucl. Phys. B 612, 59 (2001).

16. G. L. Fogli, E. Lisi, A. Marrone, A. Melchiorri, A. Palazzo, P. Serra and J. Silk, arXiv:hep-ph/0408045

17. http://www-ik1.fzk.de/tritium/ 
18. S. Eidelman et al., Phys. Lett. B592, 1 (2004).

19. J. N. Bahcall, H. Murayama and C. Pena-Garay, Phys. Rev. D 70, 033012 (2004).

20. P. Crotty, J. Lesgourgues and S. Pastor, Phys. Rev. D 69 (2004) 123007.

21. S. Hannestad, arXiv:hep-ph/0404239

22. J. Burguet-Castell, D. Casper, J. J. Gomez-Cadenas, P. Hernandez and F. Sanchez, Nucl. Phys. B 695, 217 (2004).

23. B. Richter, arXiv:hep-ph/0008222

H. Minakata and H. Nunokawa, Phys. Lett. B495, 369 (2000);

K. Dick, M. Freund, P. Huber and M. Lindner, Nucl. Phys. B598, 543 (2001);

V. D. Barger, S. Geer, R. Raja and K. Whisnant, Phys. Rev. D63, 112011 (2001).

24. M. Mezzetto, CERN-NUFACT-NOTE-60.

25. J. J. Gomez-Cadenas et al. [CERN working group on Super Beams Collaboration], arXiv:hep-ph/0105297

26. D.G. Kosharev, CERN internal report CERN/ISR-DI/74-62 (1974).

27. S. Geer, Phys. Rev. D 57, 6989 (1998) [Erratum-ibid. D 59, 039903 (1999)].

28. A. De Rujula, M. B. Gavela and P. Hernandez, Nucl. Phys. B 547, 21 (1999).

29. A. Blondel et al., Nucl. Instrum. Meth. A 451, 102 (2000).

30. M. Apollonio et al., arXiv:hep-ph/0210192

31. A. Broncano and O. Mena, Eur. Phys. J. C 29, 197 (2003).

32. A. Cervera, A. Donini, M. B. Gavela, J. J. Gomez Cadenas, P. Hernandez, O. Mena and S. Rigolin, Nucl. Phys. B 579, 17 (2000) [Erratum-ibid. B 593, 731 (2001)].

33. A. Cervera, F. Dydak and J. Gomez Cadenas, Nucl. Instrum. Meth. A451, 123 (2000).

34. R. Gandhi, C. Quigg, M. H. Reno and I. Sarcevic, Astropart. Phys. 5, 81 (1996).

35. J. Bernabeu, arXiv:hep-ph/9904474 J. Bernabeu and M.C. Banuls, Nucl. Phys. Proc. Suppl. 87, 315 (2000).

36. A. Donini, M. B. Gavela, P. Hernandez and S. Rigolin, "Neutrino mixing and CPviolation," Nucl. Phys. B574, 23 (2000).

37. A. Romanino, Nucl. Phys. B574, 675 (2000).

38. J. Burguet-Castell, M. B. Gavela, J. J. Gomez-Cadenas, P. Hernandez and O. Mena, Nucl. Phys. B 608, 301 (2001).

39. J. Burguet-Castell, M. B. Gavela, J. J. Gomez-Cadenas, P. Hernandez and O. Mena, Nucl. Phys. B 646, 301 (2002).

40. H. Minakata and H. Nunokawa, JHEP 0110, 001 (2001).

41. V. Barger, D. Marfatia and K. Whisnant, Phys. Rev. D 65, 073023 (2002).

42. G. L. Fogli and E. Lisi, Phys. Rev. D 54, 3667 (1996).

43. K. Dick, M. Freund, P. Huber and M. Lindner, Nucl. Phys. B 598, 543 (2001).

44. A. Donini, D. Meloni and P. Migliozzi, Nucl. Phys. B 646, 321 (2002).

45. D. Autiero et al., Eur. Phys. J. C 33, 243 (2004).

46. A. Donini, D. Meloni and S. Rigolin, JHEP 0406, 011 (2004).

47. A. Donini, E. Fernandez-Martinez, P. Migliozzi, S. Rigolin and L. Scotto Lavina, arXiv:hep-ph/0406132

48. V. Barger, D. Marfatia and K. Whisnant, Phys. Rev. D 66, 053007 (2002).

49. H. Minakata, H. Nunokawa and S. J. Parke, arXiv:hep-ph/0310023

50. H. Minakata, H. Nunokawa and S. J. Parke, Phys. Rev. D 68, 013010 (2003).

51. O. Mena and S. Parke, arXiv:hep-ph/0408070

52. H. Minakata, H. Sugiyama, O. Yasuda, K. Inoue and F. Suekane, Phys. Rev. D 68, 033017 (2003).

53. K. B. McConnel and M. H. Shaevitz, arXiv:hep-ex/0409028

54. K. Kodama et al., [OPERA Collaboration] CERN-SPSC-98-25. Updated information can be found at 
http://operaweb.web.cern.ch

55. O. Mena, arXiv:hep-ph/0305146

56. O. Mena et al, in preparation.

57. I. Ambats et al. [NOvA Collaboration], FERMILAB-PROPOSAL-0929.

58. Y. Itow et al., arXiv:hep-ex/0106019 